\title{
Modification of Copper (II) Ion-exchange Properties of Free- standing Alginate Membrane by Embedding with Zeolite
}

\author{
Masahide HAgIRI $^{1 *}$, Ryuya WATANABE ${ }^{1}$, Ami HIRUTA $^{1}$, and Keita KASHIMA ${ }^{2}$ \\ ${ }^{1}$ Department of Applied Chemistry and Biochemistry, Fukushima College, National Institute of Technology, Nagao 30, \\ Kamiarakawa, Taira, Iwaki, Fukushima 970-8034, Japan \\ ${ }^{2}$ Department of Materials Chemistry and Bioengineering, Oyama College, National Institute of Technology, 771 Nakakuki, Oyama, \\ Tochigi 323-0806, Japan
}

\begin{abstract}
Insolubilized alginate membranes were obtained by crosslinking alginate with calcium ions, and alginate membranes embedded with zeolite in order to improve their ion exchange capacities were also successfully prepared. The alginate membranes were ion exchangeable due to the release of crosslinking ions, and showed an ion exchange capability for copper (II) ions that was comparable to that for alginate hydrogel beads. Additionally, while both the insolubilized alginate membranes and the zeolite-embedded membrane exhibited ion-exchange abilities, additional ion-exchange capacities was imparted by embedding zeolite in the latter. However, it was also found that embedding partially inhibits the ion-exchange capacities of the zeolite contained therein.
\end{abstract}

\section{Introduction}

Although copper is one of the elements essential for living organisms, it is also an element that may adversely affect ecosystems if they are subjected to long-term exposure. In developing countries, there are areas where artificial or non-artificial pollution of underground water by copper and other heavy metals is a serious concern (Fenglian and Wang, 2011). Therefore, technological developments aimed at the recovery and removal of heavy metals from water systems is an ongoing issue for those seeking to facilitate sustainable development in industry and society.

Various conventional methods of removing ions from solutions have been applied to this problem. These include, for example, ion-exchange, evaporative concentration, chemical precipitation, reverse osmosis, adsorption, electrodialysis, and others (Barakat, 2011). Among these methods, adsorption and ion-exchange methods are considered to be effective for the removal of copper ions in low concentration solutions from the viewpoint of economy and efficiency, and various chelating ion-exchange resins have been developed and put into use for the removal of heavy metal ions (Botelho Jr. et al., 2019).

In recent years, new ion removal processes using biological materials have also been studied (Ho, 2003; Benaïssa and Elouchdi, 2007). One resource-rich biological material is the polysaccharide of brown algae, which is a marine biomass (Lee and Lee, 2016). Alginate is a polysaccharide obtained from brown and red algae that can be extracted as a soluble salt, such as sodium alginate. Alginates are well known as gel-forming materials because they form hydrogels by ionic or covalent crosslinking (Mongar and Wassermann, 1947). This feature has prompted wide-ranging studies on the formation of alginate microcapsules and their applications. Alginate microcapsules acquire various functions when combined with functional microparticles (Papageorgiou et al., 2006; Hong et al., 2016). For example, Vipin et al. (2013) prepared an alginate gel containing Prussian blue for use in the removal of radioactive cesium generated during a nuclear accident. Additionally, our recent studies revealed the potential utility of alginate membranes and showed how they could be compositely prepared using various materials (Kashima and Imai, 2012; Kashima and Imai, 2017). The membrane preparation method discussed in those studies was further applied to the development of an alginate membrane containing activated carbon that could be used efficiently for dye removal. Advantageously, this membrane could also be used for membrane separation processes (Kashima et al., 2016). Furthermore, as an application for another biopolymer, the removal of cesium ions from aqueous solutions has also studied and reported using a chitosan membrane embedded with Prussian blue (Fujisaki et al., 2019).

In this paper, we report a preparation method for alginate membranes and their resulting ion-exchange capabilities. We also report the influence of zeolite embedding on the ion-exchange capacities of these membranes.

\section{Experimental}

* Corresponding author: hagiri@fukushima-nct.ac.jp 


\subsection{Materials}

Sodium alginate (Kanto Chemical), polyethylene glycol (PEG), average molecular weight 1000 (Wako Chemical), calcium chloride (Wako Chemical), copper (II) chloride (Wako Chemical), and $\mathrm{Na}^{+}$exchanged Atype zeolite (Wako Chemical) were used as received without further purification. In every membrane preparation, experiments were performed using the same lots of sodium alginate and PEG. The particle size of the $\mathrm{Na}^{+}$exchanged A-type zeolite was in the range of 2-5 $\mu \mathrm{m}$. The copper and calcium standard solutions used for quantitative analysis were prepared from $1000 \mathrm{mg} / \mathrm{L}$ of copper (II) ion standard solution (Junsei Chemical) and $1000 \mathrm{mg} / \mathrm{L}$ calcium ion standard solution (Wako Chemical) by dilution with nitric acid. Solutions containing copper (II) ions used in the ion-exchange experiments were prepared by dissolving copper (II) chloride dihydrate (Wako Chemical) in distilled water. The $\mathrm{pH}$ of the copper (II) ion solutions used in the ionexchange experiments was within the range of 4.5-6.0.

\subsection{Preparation of Membranes}

The preparation of free-standing alginate membranes crosslinked with calcium ions was performed as follows. First, a $1.0 \%$ aqueous sodium alginate solution containing 0-3.0\% PEG was prepared and aliquoted into a polypropylene container (diameter $25 \mathrm{~mm}$ ) and then allowed to stand in an incubator to dry $\left(25^{\circ} \mathrm{C}, 48 \mathrm{~h}\right)$. PEG was added to improve the permeability of the membranes. Alginate membranes were then obtained by contacting the obtained dried product with a 10\% aqueous calcium chloride solution.

The preparation of alginate membranes containing zeolite was conducted using a method similar to that described above. Zeolite was added at $0-5.0 \%$ to a $1.0 \%$ aqueous sodium alginate solution containing $0-5.0 \%$ PEG and then well dispersed by ultrasonication. The dispersion was then dispensed into a polypropylene container and dried by heating in an oven $\left(80^{\circ} \mathrm{C}, 30 \mathrm{~min}\right)$ with an air flow, and then allowed to stand in an incubator $\left(25^{\circ} \mathrm{C}, 48 \mathrm{~h}\right)$ for additional drying. In the next step, the dried product obtained was brought into contact with a $10 \%$ aqueous calcium chloride solution in order to obtain alginate membranes containing zeolite.

The alginate beads crosslinked with calcium ions used for ion-exchange ability comparisons were prepared by a microencapsulation method in which an aqueous solution consisting of $1.0 \%$ sodium alginate and containing $0-10 \%$ PEG was dropped at a constant rate into an aqueous solution containing $10 \%$ calcium chloride in order to prepare a hydrogel containing PEG.

\subsection{Evaluation of Membranes}

Preliminarily, the membranes were washed with water at $60^{\circ} \mathrm{C}$ for 10 minutes before immersion to remove the PEG. In the batch type isothermal adsorption test, the alginate membranes or beads obtained above were immersed in a solution containing copper ions and shaken for a fixed time at a constant temperature $\left(25^{\circ} \mathrm{C}\right)$.
After immersion, the copper ion content of the solution was quantified by inductively coupled plasma optical emission spectrometry (ICP-OES; Perkin-Elmer Optima 7300). For some samples, we also investigated the calcium ion elution behavior. The concentration was determined using a calibration curve based on an external standard. The visible light transmission spectra of membranes were recorded by a Shimadzu Biospec1600 spectrophotometer.

\section{Results and Discussion}

\subsection{Preparation and evaluation of alginate membranes}

Crosslinked alginate membranes were obtained by contacting the dried sodium alginate membranes with a calcium chloride solution using the method described in our previous report (Kashima and Imai, 2017). From the results obtained, it appears that alginate has numerous crosslinking points that result in a crosslinked body, due to densification of polymeric structure by drying process. The membranes did not shrink in either the aqueous alginate solution drying process or the process of contact with the calcium chloride solution. This was confirmed by the fact that the obtained membranes had the same sizes and shapes as the containers used in their production. The transmission spectra in the visible light region for the obtained alginate membranes are shown in Figure 1. Photographs showing the appearance of the membranes are also included.

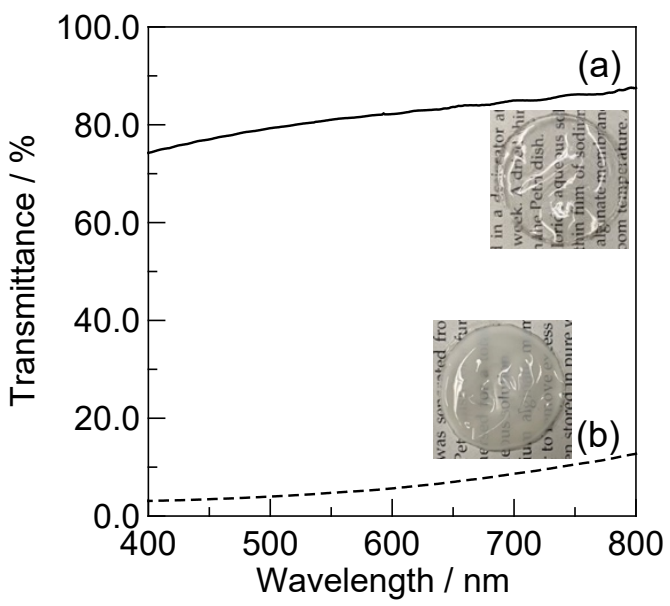

Figure 1. Visible-light transmission spectra of the obtained alginate membranes in swollen; (a, solid line) The membrane prepared without PEG; (b, dashed line) with $1.0 \%$ PEG

The membranes obtained without PEG showed high smoothness and transparency. When up to $3.0 \%$ of the raw material composition was added with PEG as an auxiliary agent, the transparency of membranes reduced while maintaining smoothness. This devitrification was due to the introduction of voids as a result of PEG acting as a template. A conceptual diagram of void formation is shown in Figure 5 in the reference (Kashima and Imai, 
2011). Furthermore, the SEM image of the membrane cross section is shown in Figure 3 in our previous report (Kashima et al., 2016). All of the obtained membranes had a mechanical strength sufficient to allow them to be subjected to pressure filtration testing without being damaged. Therefore, after the membranes were washed, they were subjected to subsequent batch type ionexchange experiments.

In those experiments, the obtained membranes were first immersed in a solution containing $4.7 \mathrm{mmol} / \mathrm{L}(300$ $\mathrm{mg} / \mathrm{L}$ ) of copper (II) ions, after which the time changes of the concentration were observed. A plot of the relative changes in concentration of copper (II) ion, $C_{t} / C_{0}[-]$ against time, $t[\mathrm{~h}]$ is shown in Figure 2. Here, $C_{0}$ represents the initial concentration $(4.7 \mathrm{mmol} / \mathrm{L})$, and $C_{t}$ $[\mathrm{mmol} / \mathrm{L}]$ represents the concentration at time $t$.

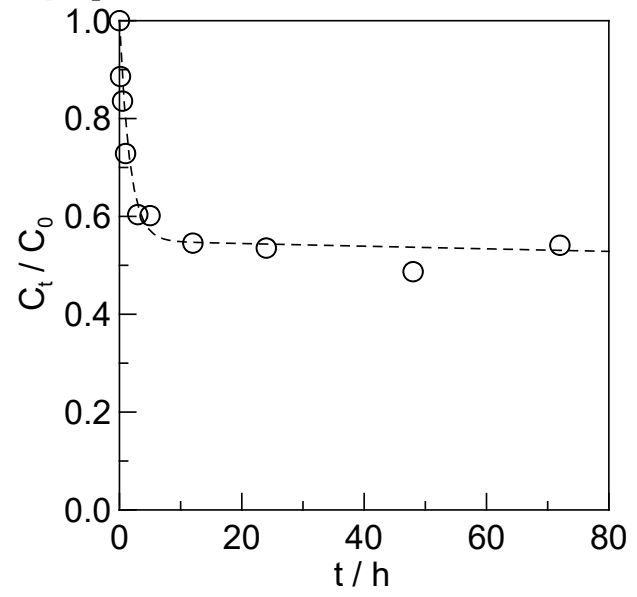

Figure 2. Time course of captured copper (II) ions onto the alginate membrane prepared without PEG.

The increase in copper (II) ion uptake was swift. In the early stage of the uptake, the increase was exponential. Its time constant was estimated to be around $1.7 \mathrm{~h}$. Chen et al. measured the exchange rate of copper (II) ions in order to apply crosslinked alginate beads to ionexchange media (Chen et al., 1997). That study reported that the ion-exchange process was fundamentally complete within about $1 \mathrm{~h}$, but also suggested the presence of a slow component of about $15 \mathrm{~h}$.

In another study, Rodrigues et al. focused on the formation kinetics of a copper ion-crosslinked alginate gel and revealed that bond formation between alginate and copper (II) ion reaches equilibrium within $30 \mathrm{~min}$ at a copper (II) ion concentration of $32 \mathrm{mmol} / \mathrm{L}$, and within about $3 \mathrm{~h}$ at $3 \mathrm{mmol} / \mathrm{L}$ (Rodrigues et al., 2006). The time constant of $1.7 \mathrm{~h}$ in the exponentially decreasing region obtained in our experiment is consistent with those results, and it is clear that the alginate in the membrane plays a role as an ion-exchange medium. Furthermore, the elution amount of calcium ions examined along with the copper (II) ion analysis was equimolar to the copper (II) ions removed. This also supports the occurrence of ion exchange by the alginate membrane. The immersion time was set to 72 hours in the batch-type ion-exchange test, which thoroughly considered the presence of the late time component that will be shown later.
Subsequently, alginate membranes prepared by adding 0-3\% PEG were subjected to ion-exchange tests using various concentrations of copper (II) ion solution $\left(\left[\mathrm{Cu}^{2+}\right]=1.6-7.9 \mathrm{mmol} / \mathrm{L}\right)$ in order to obtain an ionexchange isotherm. The solution amount and ambient temperature were constant at $10 \mathrm{~mL}$ per membrane and $25^{\circ} \mathrm{C}$, respectively. The $\mathrm{pH}$ levels of the copper (II) ion solutions used in the ion-exchange experiments were all in the range of 4.5-6.0. Figure 3 shows an adsorption isotherm in which the amount of copper (II) ions trapped per unit mass of alginate $q[\mathrm{mmol} / \mathrm{g}]$ is plotted against the equilibrium concentration $C[\mathrm{mmol} / \mathrm{L}]$ of the copper (II) ions. The ion-exchange amount at equilibrium estimated from the figure is approximately $2.0 \mathrm{mmol} / \mathrm{g}$. This corresponds to $30 \mu \mathrm{mol} / \mathrm{memb}$. and $6 \mu \mathrm{mol} / \mathrm{cm}^{2}$ for the ion-exchange amount per membrane and per membrane area, respectively.

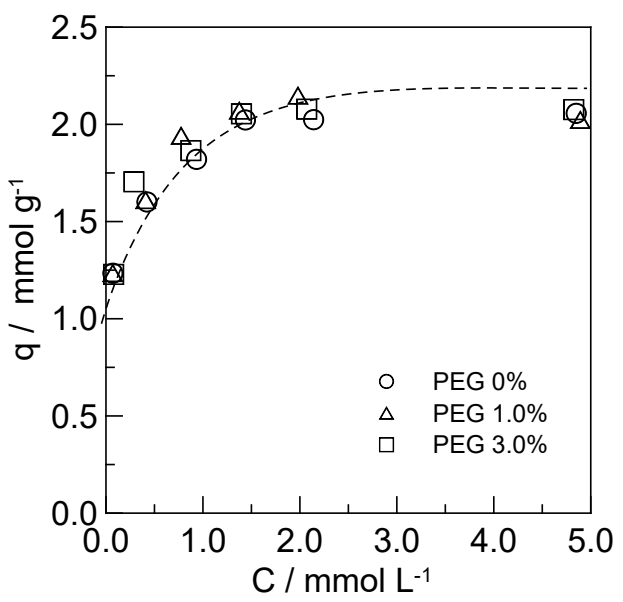

Figure 3. Isothermal plots for capturing copper (II) ions onto alginate membranes prepared with various PEG mass fractions

The ion-exchange amount increases with the equilibrium concentration and tends to saturate at high concentration, and the isotherm is a typical Langmuirtype curve (Langmuir, 1918). The linear form of the Langmuir equation, which is a typical ion-exchange and adsorption model, is shown in Eq. (1).

$$
\frac{C}{q}=\frac{1}{q^{\infty} K}+\frac{C}{q^{\infty}}
$$

Here, $C$ represents the equilibrium concentration $[\mathrm{mmol} / \mathrm{L}], q$ represents the ion-exchange amount per unit mass $[\mathrm{mmol} / \mathrm{g}], \mathrm{K}$ represents the adsorption equilibrium constant $[\mathrm{L} / \mathrm{mmol}]$, and $q^{\infty}$ represents the saturated ionexchange amount $[\mathrm{mmol} / \mathrm{g}]$. Since the experimental results fit the Langmuir-type adsorption isotherm, the results are analyzed using Eq. (1). The plot of $C / q$ vs. $C$ in Eq. (1), which is the so-called Langmuir plot, is shown in Figure 4. The dotted line in the figure presents an approximately straight line for the Langmuir plot of a free-standing membrane containing 3.0\% PEG in a raw solution. From these results, it was found that the freestanding membrane exhibits a copper ion-exchange capability and that its Langmuir plot is linear. 


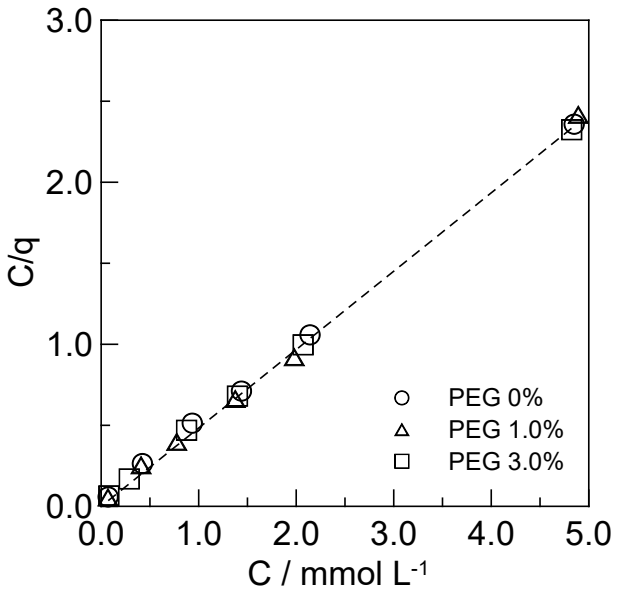

Figure 4. Isothermal plots for adsorption of copper (II) ions onto alginate membranes adapted for Langmuir model

Table 1 shows the relationship between the amounts of PEG used in alginate membrane preparations and the copper ion-exchange capacity obtained from the Langmuir plot. Similar results were obtained from ionexchange isothermal experiments of alginate beads, and those results are also shown. Here, $q_{m}{ }^{\infty}$ represents the ion-exchange capacity per membrane, and $q^{\infty}$ represents the ion-exchange capacity per membrane as a dried body. Table 1 also shows that the introduction of PEG into the membrane does not affect its copper ion-exchange capacity. Additionally, it was found that the membrane has an ion-exchange capacity equivalent to alginate beads. Alginate beads are a hydrogel containing a large amount of water, and the mass fraction of alginate in beads prepared from sodium alginate solution is approximately $1 \mathrm{w} / \mathrm{v} \%$.

In contrast, the obtained free-standing membrane is denser than gel beads, and the alginate volume fraction is about $7-8 \mathrm{w} / \mathrm{v} \%$. Although the diffusivity of the solvent and the ions into the material structure is considered to be different, it is interesting to note that the copper ionexchange capacities of the beads and membranes were nearly equal.

Table 1. Estimated copper (II) ion-exchange capacity of alginate membranes.

\begin{tabular}{ccr}
\hline Mass percentage & \multicolumn{2}{c}{$\mathrm{Cu}^{2+}$ ion-exchange capacities } \\
\cline { 2 - 3 } of PEG [\%] & $q_{m}{ }^{\infty}[\mathrm{mmol} / \mathrm{memb}]$. & $q^{\infty}[\mathrm{mmol} / \mathrm{g}]$ \\
\hline $0^{\mathrm{a}}$ & - & 1.90 \\
0 & 0.0278 & 2.09 \\
0.30 & 0.0291 & 2.19 \\
0.50 & 0.0273 & 2.05 \\
1.0 & 0.0277 & 2.08 \\
3.0 & 0.0287 & 2.16 \\
\hline & \multicolumn{2}{c}{}
\end{tabular}

\subsection{Preparation and evaluation of alginate membranes embedded with zeolite}

Insoluble solid substances present in an aqueous sodium alginate solution during the preparation of alginate beads are easily incorporated into the gel. This method is known as microencapsulation and is currently used in the production of persistent pesticides, medicines, and other products. The alginate membranes prepared in this study were also capable of incorporating solid matters into their compositions. For example, fine particles of activated carbon have been introduced into membranes that can adsorb to organic dyes (Kashima et al., 2016). Focusing on this point, we are now proceeding with the preparation of membranes containing an adsorbent and determining their adsorption capacities.

The above examinations revealed that it is possible to remove copper (II) ions with alginate membranes. We further realized that by introducing a cation-exchange medium into this membrane, it would be possible to enhance its ion-exchange capacity. Therefore, a method of introducing zeolite, which is an inorganic material having an ion-exchange capacity, into the membrane was explored. In this study, A-type zeolite with sodium ions as a charge compensating cation was used. A-type zeolite, which is known as Linde Type A (LTA) in the structural code, has a structure in which the basic framework sodalite cages are connected (Reed and Breck, 1956). The pore size is $0.41 \mathrm{~nm}$, and the composition of the unit cell is represented by $\mathrm{Na}_{12}\left[\mathrm{Al}_{12} \mathrm{Si}_{12} \mathrm{O}_{48}\right] \cdot 96 \mathrm{H}_{2} \mathrm{O}$. Since the sodium ions used as charge compensating cations are exchangeable, A-type zeolite is ion-exchange capable and is currently used in various fields such as industrial production, agriculture, and toiletry products.

In the preparation of alginate membranes containing zeolite, an aqueous sodium alginate solution in which zeolite was added and dispersed by ultrasonic irradiation was used as a raw material. However, when the solution was prepared the same way as used for membranes without zeolite, body cracks frequently occurred during the drying process. One of the causes for this is the aggregation and precipitation of zeolite during the slow drying process. To avoid this, it was thought that quick drying would be more appropriate. However, rapid drying leads to the formation of cracks, and insufficient drying leads to embrittlement. Therefore, the drying process was divided into two steps: rapid drying under initial heating, followed by slow drying at room temperature. The former was $80^{\circ} \mathrm{C}$ for 30 minutes under air convection, and the latter was approximately $25^{\circ} \mathrm{C}$ for 24 hours. These conditions significantly decreased dried body crack occurrences, and the products thus obtained were then insolubilized by immersion in an aqueous calcium chloride solution.

The mass fractions of alginate $M F_{A}$ and zeolite $M F_{Z}$ are defined by the following Eq. (2). Here, $W_{A}$ is the mass of alginate in the membrane as solid content, and $W_{Z}$ is the zeolite.

$$
M F_{A}=\frac{W_{A}}{W_{A}+W_{Z}}, M F_{Z}=\frac{W_{Z}}{W_{A}+W_{Z}}
$$

Membranes containing various amounts of zeolite were prepared with a maximum $M F_{Z}$ of 0.83 . The mass fraction of zeolite $M F_{Z}=0.83$, which corresponds to $5 \%$ in the feed casting solution. In addition, stable membranes were obtained when the amount of PEG 
added was $0-3.0 \%$. Hereinafter, the added amounts of zeolite and PEG are $5.0 \%\left(M F_{Z}=0.83\right)$ and $1.0 \%$ for all cases. Photographs showing the appearance of alginate membranes embedded with zeolite at $M F_{Z}=0.83$ are provided in Figure 5.

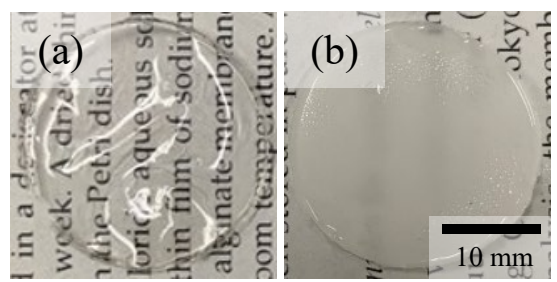

Figure 5. Photographs of alginate membrane;

(a) prepared without PEG in the same manner as shown in Figure 1a; (b) embedded with zeolite, $M F_{Z}=0.83$

The alginate membranes embedded with zeolite were subjected to the same ion-exchange test described above in order to obtain ion-exchange isotherm values. One membrane was immersed in $10 \mathrm{~mL}$ of the solution during each experiment. The amount of zeolite added to the membrane in the composition of raw solutions was $3 \%$, which amounts to three times the alginate content of the membrane. Figure 6 shows an adsorption isotherm in which the amount of copper (II) ion trapped per unit mass of alginate $q[\mathrm{mmol} / \mathrm{g}]$ is plotted against the equilibrium concentration $C[\mathrm{mmol} / \mathrm{L}]$ of the copper (II) ions.

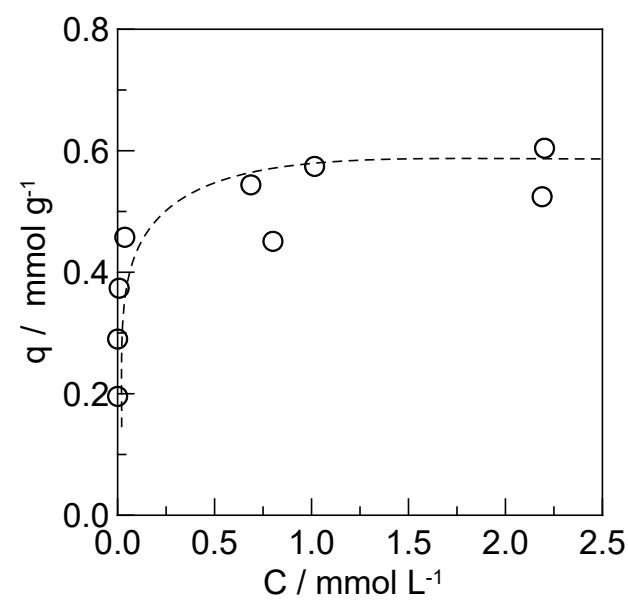

Figure 6. Isothermal plot for capture of copper (II) ions by an alginate membrane embedded with zeolite

In the case of a zeolite-embedded membrane, the crosslinking points in alginates and the framework cations of the zeolite should serve as ion-exchange sites. The plot also shows a linear relationship between $C / q$ and $C$. Therefore, the ion-exchange capacity could be simply determined from the slope of this plot. Table 2 shows the relationship between the amount of embedded zeolite and the copper ion-exchange capacity, $q_{m}{ }^{\infty}$ and $q^{\infty}$, obtained from the Langmuir plot.
Table 2. Estimated copper (II) ion-exchange capacity of alginate membranes embedded with zeolite

\begin{tabular}{lcr}
\hline \multicolumn{1}{c}{$M F_{Z}[-]$} & \multicolumn{2}{c}{$\mathrm{Cu}^{2+}$ ion-exchange capacity } \\
\cline { 2 - 3 } & $q_{m}{ }^{\infty}[\mathrm{mmol} / \mathrm{memb}]$. & $q^{\infty}[\mathrm{mmol} / \mathrm{g}]$ \\
\hline $1.00^{\mathrm{a}}$ & - & 3.21 \\
$0^{\mathrm{b}}$ & 0.0277 & 2.08 \\
$0.83^{\mathrm{c}}$ & 0.0515 & 0.585 \\
\hline
\end{tabular}

As a result of this analysis, it was found that the introduction of zeolite improved the ion-exchange capacity per membrane by about double. Additionally, while alginate naturally possesses ion-exchange capabilities, when it is combined with zeolite, the ionexchange capacity of the membrane improves. Therefore, it can be said that the addition of zeolite contributes to an improvement in the overall ion-exchange capacity of the membrane. On the other hand, focusing on the ionexchange capacity per mass, it appears that the value can be as small as $0.585 \mathrm{mmol} / \mathrm{g}$. The ion-exchange capacity per mass was $3.21 \mathrm{mmol} / \mathrm{g}$ for the zeolite used, and 2.08 $\mathrm{mmol} / \mathrm{g}$ for the alginate membrane without zeolite. If the membrane structure does not inhibit these ion exchanges, the ion-exchange capacity $I E C_{A Z}$ of the zeolite composite membrane is estimated using Eq. (3) below based on the solid composition of the membrane. Here, $I E C_{A}$ and $I E C_{Z}$ are the ion-exchange capacities $[\mathrm{mmol} / \mathrm{g}]$ of alginate and zeolite, respectively.

$$
I E C_{A Z}=I E C_{A} \cdot M F_{A}+I E C_{Z} \cdot M F_{Z}
$$

The $I E C_{A Z}$ calculated using the values shown in Table 2 is $3.02 \mathrm{mmol} / \mathrm{g}$. Since the experimental value for the actual membrane is far below this, it is considered likely that inhibition mechanisms are involved. More specifically, it is thought that both a decrease in the crosslinking point of alginate acting as an ion-exchange site and an inhibition of the zeolite ion-exchange capability due to clathration are involved. Since $M F_{A}$ is smaller than $M F_{Z}$, and $I E C_{A}$ is smaller than $I E C_{Z}$, the influence of the reduction in the number of crosslinks is considered to be small as well. This means that the latter has a significant impact on quantity. Therefore, the inhibition of the zeolite ion-exchange capability is linked to the decrease in the ion diffusion rate caused by the alginate pore blockage and the increase in the ion concentration around it. This is supported by the fact that the adsorption constant $K$ in the Langmuir plot of the membrane is smaller than both the alginate membrane and the zeolite powder.

As significant findings, Vipin et al. (2013) prepared alginate beads embedded with Prussian blue for removing cesium ions from contaminated water (Vipin et al., 2013) and then demonstrated an efficient process for the removal of these ions. Meanwhile, Yurekli et al. (2016) prepared zeolite nanoparticle impregnated polysulfone membranes for heavy metal ion removal (Yurekli, 2016). This material also showed a high removal performance for heavy metal ions. In this way, ion-exchange performance evaluations have been 
performed previously on composite gels and membranes in which various adsorbents have been embedded. However, ion-exchange performance comparisons between the membranes with and without embedded substances have not been conducted previously. Hence, this study is expected to provide essential insights into the issues involved in the preparation and application of such composite materials.

\section{Conclusion}

In order to facilitate the development of materials with low environmental impact, this study examined the copper ion-exchange capabilities of alginate membranes with and without embedded zeolite. The results obtained show that both membrane types exhibited ion-exchange capacities and that increased ion-exchange capacity could be obtained by embedding zeolite in the membranes. However, it was also found that the embedding partially inhibited the ion-exchange capability of the zeolite. As a result, we are currently studying the relationship between the amount of zeolite added and its effect on ion-exchange capacity. Experiments are also underway to permeate the feed solution through a membrane that has improved permeability by the addition of PEG.

\section{Acknowledgments}

This study was supported by the research grant from the Maeda Engineering Foundation in Japan. The authors would like to thank N. Usuba, C. Sagawa, and R. Sakamoto for their technical assistance in our experiments.

\section{References}

Fenglian, F. and Q. Wang; "Removal of Heavy Metal Ions from Wastewaters: A Review," J. Environ. Manage., 92, 407-418 (2011)

Barakat, M. A.; "New Trends in Removing Heavy Metals from Industrial Wastewater," Arab. J. Chem., 4, 361-377 (2011)

Botelho Jr., A. B., D. B. Dreisinger, and D. C. R. Espinosa; "A Review of Nickel, Copper, and Cobalt Recovery by Chelating Ion-exchange Resins from Mining Processes and Mining Tailings," Min. Metall. Explor., 36, 199-213 (2019)

Ho, Y.-S.; "Removal of Copper Ions from Aqueous Solution by Tree Fern," Water Res., 37, 2323-2330 (2003)

Benaïssa, H. and M. A. Elouchdi; "Removal of Copper Ions from Aqueous Solutions by Dried Sunflower Leaves," Chem. Eng. Process., 46, 614-622 (2007)

Lee, O. K. and E. Y. Lee; "Sustainable Production of Bioethanol from Renewable Brown Algae Biomass," Biomass Bioenerg., 92, 70-75 (2016)

Mongar, J. L. and A. Wassermann; "Ionic Exchange and Fibre Contraction," Nature, 159, 746 (1947)
Papageorgiou, S. K. , F. K. Katsaros, E. P. Kouvelos, J. W. Nolan, H. L. Deit, and N. K. Kanellopoulos; "Heavy Metal Sorption by Calcium Alginate Beads from Laminaria digitata," J. Hazard. Mater., 137, 1765-1772 (2006)

Hong, H.-J., J. Ryu, I.-S. Park, T. Ryu, K.-S. Chung and B.-G. Kim; "Investigation of the Strontium ( $\mathrm{Sr}(\mathrm{II})$ ) Adsorption of an Alginate Microsphere as a Low-cost Adsorbent for Removal and Recovery from Seawater," $J$. Environ. Manage., 165, 263-270 (2016)

Vipin, A. K., B. Hu and B. Fugetsu; "Prussian Blue Caged in Alginate/Calcium Beads as Adsorbents for Removal of Cesium Ions from Contaminated Water," $J$. Hazard. Mater., 258-259, 93-101 (2013)

Kashima, K. and M. Imai; "Impact Factors to regulate Mass Transfer Characteristics of Stable Alginate Membrane Performed Superior Sensitivity on Various Organic Chemicals,” Procedia Eng., 42, 964-977 (2012)

Kashima, K. and M. Imai; "Selective Diffusion of Glucose, Maltose, and Raffinose through Calcium Alginate Membranes Characterized by a Mass Fraction of Guluronate," Food Bioprod. Process., 42, 213-221 (2017)

Kashima, K. and M. Imai; "Dominant Impact of the $\alpha$-LGuluronic Acid Chain on Regulation of the Mass Transfer Character of Calcium Alginate Membranes," Desalin. Water Treat., 34, 257-265 (2011)

Kashima, K., K. Osawa, M. Hagiri and M. Imai; "High Performance Dye Removal by Embedded Activated Carbon in Calcium Alginate Membrane Combination with Low-Molecular-Weight Polyethylene Glycol," Proceedings of the 5th Asian Conference on Innovative Energy and Environmental Chemical Engineering, pp. 70-73, Yokohama, Japan (2016)

Fujisaki, T., K. Kashima, M. Hagiri and M. Imai; "Isothermal Adsorption Behavior of Cesium Ions in a Novel Chitosan-Prussian Blue Based Membrane," Chem. Eng. Technol., 42, 910-917 (2019)

Chen, J., F. Tendeyong and S. Yiacoumi; "Equilibrium and Kinetic Studies of Copper Ion Uptake by Calcium Alginate," Env. Sci. Technol., 31, 1433-1439 (1997)

Rodrigues, J. R. and R. Lagoa; "Copper Ions Binding in Cu-Alginate Gelation," J. Carbohydr. Chem., 25, 219232 (2006)

Langmuir, I.; "The Adsorption of Gases on Plane Surfaces of Glass, Mica and Platinum," J. Am. Chem. Soc., 40, 1361-1403 (1918)

Reed, T. B. and D. W. Breck; "Crystalline Zeolites. II. Crystal Structure of Synthetic Zeolite, Type A," J. Am. Chem. Soc., 78, 5972-5977 (1956)

Yurekli, Y.; "Removal of Heavy Metals in Wastewater by Using Zeolite Nano-Particles Impregnated Polysulfone Membranes," J. Hazard. Mater., 309, 53-64 (2016) 